\title{
Complications of Ultrasound Guided Renal Biopsy in 100 Children: Experience at a Tertiary Care Hospital
}

\author{
Mohammed Maruf-Ul-Quader ${ }^{1 *}$ \\ Susmita Biswas ${ }^{1}$ \\ Mitra Datta ${ }^{2}$ \\ Muhammad Jabed Bin Amin Chowdhury ${ }^{2}$ \\ Salina Haque ${ }^{2}$ \\ Kazi Mohammad Moinuddin ${ }^{3}$ \\ Sunanda Sen ${ }^{3}$ \\ Sawrav Deb Bappy ${ }^{2}$

\footnotetext{
Department of Pediatric Nephrology Chittagong Medical College

Chattogram, Bangladesh.

${ }^{2}$ Department of Pediatrics

Chittagong Medical College

Chattogram, Bangladesh. Chittagong Medical College

Chattogram, Bangladesh.
} \\ ${ }^{3}$ Department of Radiology and Imaging
}

\begin{abstract}
Background: Use of automated device with ultrasound guidance in renal biopsy has improved the adequacy and reduced the complication. Chittagong Medical College Hospital (CMCH) is a tertiary teaching hospital where Pediatric Nephrology Department started its journey on $11^{\text {th }}$ December 2013. Since then renal biopsy is going on. Aim of the study is to see the rate of adequacy and complication of renal biopsy along the course of years.

Materials and methods: This is a retrospective study carried on 100 consecutive ultrasonography guided percutaneous renal biopsy from lower pole of left native kidney performed by the pediatric nephrologist. All hospitalized children aged up to 12 years admitted since 2014 were included. Spring loaded automated biopsy needle was used in $88 \%$ cases and Trucut biopsy needle in $12 \%$ cases.

Results: Most of the patients were aged between 1 to 10 years with male female ratio 0.9:1. Midazolam was used for sedation in all patients except one who needed general anesthesia. Light microscopy and Direct Immunofluorescence (DIF) report was made but no facility for electron microscopy. Gross hematuria was experienced in 5\% cases and one case needed blood transfusion. There was one case with blood clot in urinary bladder causing dysuria but no urinary retention was observed. Post biopsy perinephric hematomas developed in 4 cases. Number of needle passes to obtain adequate biopsy material in native kidney was $\leq 3$ in $78 \%$ cases. Adequate specimen was found in $95 \%$ cases.
\end{abstract}

Conclusion: Percutaneous renal biopsy can be done adequately and safely in resource poor setup if pediatric nephrologist and radiologists are available.

Key words: Biopsy; Hematuria; Hematoma.

\section{INTRODUCTION}

Kidney biopsy is an invasive but irreplaceable procedure for the nephrologists ${ }^{1}$. Despite introduction of more advanced molecular biology, techniques, renal biopsy is still a gold standard tool for determining diagnosis, prognosis and treatment ${ }^{2}$.

The first closed or percutaneous renal biopsy was reported by Ball in $1934^{3}$. Clinical use of this technique routinely introduced in the 1950s. There after advancements have been made in biopsy technique to improve diagnostic yield while minimizing complication. In 1962 the use of radiological images was introduced for the localization of the kidney, later replaced by the ultrasound real-time imaging ${ }^{4}$

In renal biopsy, use of automated device and ultrasound guidance improved the adequacy and reduced the complication ${ }^{5}$. Although minor consequences occur more frequently but major complication occur rarely. The success of percutaneous renal biopsy is determined both by the adequacy of the tissue and the incidence of complications. Gross or macroscopic hematuria is the most frequent important major complication if it necessitates blood transfusion, surgical intervention/embolization or prolonged hospital stay ${ }^{6,7}$. 
Infection after kidney biopsy has been described in some case series, but if sterile technique is used and unless bowel perforation occur, it is rare. For this reason, careful evaluation of risks and benefits must be taken into account, and all measures to minimize the risk of complications must be observed ${ }^{1}$.

Complications of renal biopsy tend to vary from center to center and are also affected by several clinical and technical factors. Chittagong Medical College Hospital $(\mathrm{CMCH})$ is a tertiary teaching hospital where Department of Pediatric Nephrology started its journey on 11th December 2013. Since then renal biopsy is going on. So aim of the study is to see the frequency of complications and adequacy of renal biopsy along the course of years.

\section{MATERIALS AND METHODS}

This is a retrospective study carried on 100 consecutive ultrasonography guided percutaneous renal biopsy from lower pole of left native kidney since 2014 by pediatric nephrologist with a 16 needles under adequate sedation. All were hospitalized cases aged upto 12 years. Biopsy procedures were performed in the Radiology and Imaging Department of $\mathrm{CMCH}$ after taking proper consent. I/V cannula was kept in situ for emergency medication. Maximum aseptic precaution was maintained and $2 \%$ lignocaine was used for local anesthesia. All biopsies were done under oral antibiotic coverage as total aseptic procedure was difficult to maintain. Two cores of tissues were obtained: one of them was kept in normal saline for immunofluorescence microscopy and another one in formalin for light microscopy. Pressure dressing was applied on biopsy site with binder for adequate hemostasis. Vitals were followed up for a period of 24 hours after the procedure. Three consecutive urine samples were observed in clear plastic jar to identify the presence of gross hematuria. Paracetamol suppository was given to every patient to reduce pain and advised as required for further pain management. Next day, routine post biopsy ultrasonography was done to evaluate the presence of subscapular bleeding or perinephric hematoma. Complications were categorized as major or minor. Major complications included need for blood transfusion, surgical intervention like nephrectomy, angioembolization, bladder obstruction and death related to the procedure. Minor complications were defined as transient gross hematuria or minor hematoma reported by follow up USG that resolved spontaneously and did not require any transfusions or surgical intervention $^{8}$. Adequacy of renal tissue was defined as renal tissue containing $\geq 5$ glomeruli and histopathologist being able to reach a diagnosis based on the sampled tissue ${ }^{9,10}$. Information regarding name, age, sex, weight of the patient, indication for biopsy, type of sedation used, size of biopsy needle, name of the pediatric nephrologist, radiologist, assisting doctor and nurse, number of needle passes, complication details (If any) post biopsy USG findings, duration of hospital stay.

\section{RESULTS}

A total 100 ultrasound-guided renal biopsy was performed during the course of six consecutive years. Demographic data (Table I) showing age of maximum patients (65\%) ranged 1-10 years. Only two patients were below 2 years and youngest one was 8 months old ((Figure 1). Sex distribution showing M:F0.9:1. Midazolam was used for sedation in all patients except one needed general anesthesia. Spring loaded automated biopsy needle was used in $88 \%$ cases and $12 \%$ cases were done by Trucut biopsy needle. Frequency of renal biopsy during the course of time since 2014 was depicted in (Figure 2). Majority of the procedures were performed in the year 2019. Adequate renal tissue was obtained for diagnosis in $95 \%$ cases. In $78 \%$ cases maximum number of needle passes to kidney was $\leq 3$ to obtain adequate biopsy material. The major complication rate was $2 \%$ and minor complication $8 \%$ (Table II).

Table I : Age and sex distribution.

\begin{tabular}{|l|c|}
\hline$<1$ year & $2 \%$ \\
\hline 1 to 10 year & $65 \%$ \\
\hline$>10$ year & $33 \%$ \\
\hline Male: Female & $0.9: 1$ \\
\hline
\end{tabular}

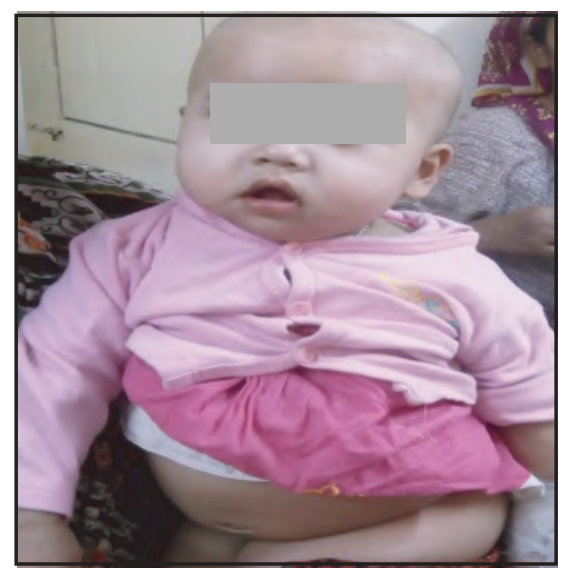

Figure 1 : Youngest one- 8 months old.

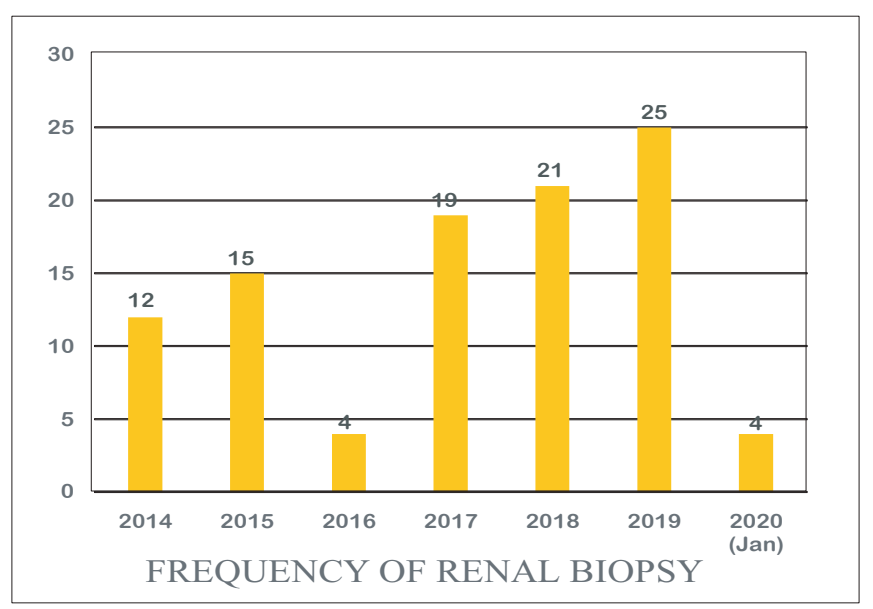

Figure 2 : Freuency of renal biopsy since 2014 . 
Table II : Complications and adequacy of renal biopsy.

$$
\text { Complications and Adequacy }
$$

\section{Major Complications}

Gross hematuria and blood transfusion required

Blood clot in urinary bladder with dysuria

\section{Minor Complications}

Gross hematuria

Post biopsy perinephric hematomas (Upto $4 \mathrm{~cm}$ ).

Adequate Specimen

Number of needle passes to obtain an

adequate biopsy material $(\leq 3)$

\section{DISCUSSION}

Despite being a common procedure in pediatric nephrology there is considerable variation in complication of renal biopsy in different centers. There is no universally agreed standard for renal biopsy. The study analyzed 100 renal biopsies performed in this center under ultrasound guidance. The overall major complication was observed in $2 \%$ cases which is similar to British Association of Pedeatric Nephrology (BAPN) standard $(\leq 5 \%)$. The UK audit by Hussain F et al found major complications in $10.4 \%$ cases where in only 3 out of the 11 centers achieved the target of $<5 \%$ and Kandaswamy $\mathrm{P}$ et al in UK found 3.2\%. Rasheed S.A.Al et al reported higher frequency of serious complication with death of one patient secondary to intestinal perforation and development of arteriovenous fistula in two patients ${ }^{10-13}$. In this study there was no death or nephrectomy or any other intervention after biopsy.

This study observed minor complication in $8 \%$ cases. Transient gross hematuria developed in 4 children which resolved spontaneously within 24 hours. However one patient with gross hematuria needed blood transfusion due to shock. These observation is similar to the study done by Sinha R. et al and incidence of gross hematura was $6 \%$ and $1 \%$ required prolonged monitoring, necessitating extension of hospital stay. Incidence was less reported by Varnell C D et al and Tondel C.et al and proportion was $3.5 \%$. and $1.7 \%$ respectively ${ }^{7,14,6}$. Reported need for blood transfusion after renal biopsy was $0.9 \%$. Rasheed S. A. Al et al observed frequent gross haematuria $(26 \%)$ after biopsy in children and 5 out of 32 children with gross hematuria required blood transfusion ${ }^{6-13}$.

In this study 4 children developed perinephric hematomas who were asymptomatic and were detected by routine post biopsy ultrasonographic observation. The size ranged up to $4 \mathrm{~cm} \times 4 \mathrm{~cm}$

which resolved spontaneously without any intervention. A systematic review and meta-analysis by Varnell C D et al on 23 studies of 5504 biopsies showed the incidence of hematoma after biopsy $11 \%-18 \%$. Kersnik L et al and other authors reported that the majority of hematomas are less than $2 \mathrm{~cm}$, and about $1 \%-2 \%$ are symptomatic. Norwegian study by Tondel $\mathrm{C}$ et al observed higher frequency of hematoma in children $(8.1 \%)$ in post biopsy routine ultrasonographic evaluation of the 715 children, one needed transfusion and another needed embolization/surgical intervention ${ }^{14,15,6}$. They also observed post-biopsy hematomas greater than $2 \mathrm{~cm}$ is likely to have clinical significance. Printza $\mathrm{N}$ et al also reported $11 \%$ subscapular hematoma and none of them needed blood transfusion ${ }^{16}$.

Reported incidence of bladder obstruction after renal biopsy in children was $0.3 \%{ }^{2}$. In this study, one patient developed dysuria due to blood clot in urinary bladder which was treated by bladder irrigation and there was no retention. But Franke $M$ et al studied 295 renal biopsy and reported one case with blood clot in urinary bladder which needed cystoscopic removal of bladder $\operatorname{clot}^{17}$

We recorded $\leq 3$ passes to obtain an adequate biopsy material in $78 \%$ cases similar to standard ( $\leq 3$ pass in $80 \%$ ) although there was no dissecting microscope in our procedure room ${ }^{11,18}$. Hussain $\mathrm{F}$ et al achieved the standard for the number of needle passes in $86.4 \%$ cases $^{10}$. Whereas the Norwegian study and Sinha R. et al reported adequate biopsy material by $\leq 2$ attempt in $85 \%$ and 72 cases respectively ${ }^{6,7}$.

BAPN standard for adequacy is $>95 \%$. Hussain $\mathrm{F}$ et al achieved the adequacy in $97.5 \%$ cases keeping the cut off number of glomeruli $\geq 10$ or limited number of glomeruli using that clear diagnosis made by the histopathologist $\mathrm{t}^{11,10}$. This study set the adequacy of renal tissue as renal tissue containing $\geq 5$ glomeruli and histopathologist being able to reach a diagnosis based on the sampled tissue ${ }^{9,10}$. With this, adequacy was achieved in $95 \%$ cases. Printza $\mathrm{N}$ et al showed the adequacy rate $97.7 \%$ and they set the adequacy at the glomeruli no $5-10^{16}$.

\section{LIMITATIONS}

i) Small sample size.

ii) Light microscope during procedure was not available to ensure glomerulus in procedure room.

iii) No facility for electron microscopy.

\section{CONCLUSION}

Percutaneous renal biopsy can be done adequately and safely in resource poor setup if pediatric nephrologist and radiologist are available.

\section{DISCLOSURE}

All the authors declared no competing interest. 


\section{REFERENCES}

1. Visconti L, Cernaro V, Ricciardi CA. Renal biopsy: Still a landmark for the nephrologist. World J Nephrol. 2016;5(4):321-327.

2. Hogan JJ, Mocanu M, Berns JS. The native kidney biopsy update and evidence for best practice. Clin J Am SocNephrol. 2016; 11:354-362.

3. Dodge WF, Daeschner CW, Brennan JC, Rosenberg HS, Travis LB, Hopps HC. Percutaneous renal biopsy in children. Official journal of the American Academy of pediatrics.1962;30(2);287-296;.http://pediatrics.aappublications.org/content/30/2/287.

4. Tape TG, Wigton RS, Blank LL, Nicolas JA. Procedural skills of practicing nephrologists. A national survey of 700 members of the American College of Physicians. Ann Intern Med. 1990; 113:392-397.

5. Kajawo S, Moloi MW, NoubiapJJ.Incidence of major complications after percutaneous native renal biopsies in adults from low-income contries to middle- income countries: A protocol for systematic review and meta-analysis.BMJ open 2018;8:e020891.

6. Tøndel C, Vikse B E, Bostad L, Svarstad E. Safety and complications of percutaneous kidney biopsies in 715 children and 8573 adults in Norway 1988-2010 Clin J Am SocNephrol. 2012; 7(10): 1591-1597.

7. Sinha R, Maji B, MeurS. A prospective audit of complications in 100 consecutive pediatric percutaneous renal biopsies done under real-time ultrasound guidance Indian J Nephrol. 2016;26(5):329-334.

8. Luciano RL and Moeckel GW. Update on the native kidney biopsy: Core curriculum 2019. Am J Kidney Dis.2019;73(3):404-415.

9. Millar M, Gooden M, Shah D, Soyibo AK, Williams J, Barton. Renal Biopsy Findings in Jamaican Children. West indian Med J. 2010;59(3):325.

10. Hussain F. Mallik M, Marks SD, Watson A R.Renal biopsies in children: Current practice and audit of outcomes.Nephrol Dial Transplan.2010;25(2):485-489.

11. [Last accessed $2020 \mathrm{Apr} 9]$ Available from: http://www.renal.org/docs/default-source/special-interest-groups/bapn/clinical-standards/renalbiosy-standards-statement.pdf?sfvrsn=2

12. KandaswamyP,Hegde S, Jeganathan et al. Audit on the practice of kidney biopsies in children. https;//dx.doi.org/10.1136/archdischild. 2012;301885:384

13. Al Rasheed S.A, Mugeiren M.M, Elidrissy ATH. The outcome of percutaneous renal biopsy in children: An analysis of 120 consecutive cases. PediatrNephol. 1990; 4:600-603.

14. Varnell CD, Stone HK, Welge JA. Bleeding Complications after Pediatric Kidney Biopsy: A Systematic Review and Meta-Analysis. CJASN. 2019; 14 (1): 57-65.

15. Kersnik T, Kenig A, Buturovi P J, Ferluga D, Avgustin C M, Kenda RB: Real-time ultrasound-guided renal biopsy with a biopsy gun in children: Safety and efficacy. ActaPaediatr. 2001;90:1394-1397.

16. Printza N, Bosdou J, Pantzaki A, Badouraki M, Kollios K, Ghogha Ch, Papachristou F. Percutaneous ultrasound-guided renal biopsy in children: A single centre experience. Hippokratia. 2011;15(3):258-261.

17. Franke M, Kramarczyk A, Taylan C, Maintz D, Hoppe B, Koerber F. Ultrasound-Guided Percutaneous Renal Biopsy in 295 Children and Adolescents: Role of Ultrasound and Analysis of Complications.2014 PLoS ONE. 9(12):e114737.

18. Hussain F, Watson AR, Hayes J. Standards for renal biopsies: Comparison of inpatient and day care procedure. PediatrNephrol. 2003;18:53-56. 